\title{
Creating a Controlled Vocabulary for the Ethics of Human Research: Towards a Biomedical Ethics Ontology
}

\author{
David Koepsell \\ Delft University of Technology
}

ROBERT ARP

National Center for Biomedical Ontology

JENNIFER Fostel

National Institutes of Health

\section{BARRY SMITH}

State University of New York at Buffalo and National Center for Biomedical Ontology

ABSTRACT: ONTOLOGIES DESCRIBE REALITY IN SPECIFIC domains in ways that can bridge various disciplines and languages. They allow easier access and integration of information that is collected by different groups. Ontologies are currently used in the biomedical sciences, geography, and law. A Biomedical Ethics Ontology (BMEO) would benefit members of ethics committees who deal with protocols and consent forms spanning numerous fields of inquiry. There already exists the Ontology for Biomedical Investigations (OBI); the proposed BMEO would interoperate with OBI, creating a powerful information tool. We define a domain ontology and begin to construct a BMEO, focused on the process of evaluating human research protocols. Finally, we show how our BMEO can have practical applications for ethics committees. This paper describes ongoing research and a strategy for its broader continuation and cooperation.

KEY WORDS: ontology, taxonomy, ethics committee review, automation, semantics

Received: August 6, 2008; revised: February 4, 2009

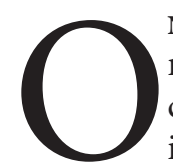
NE OF THE MOST PRESSING problems facing research ethics committees (RECs or "ethics committees" herein) is an overabundance of information. As with any bureaucracy faced with changing rules and requirements, as well as a constant stream of new cases, committee members barely have time to keep up. There would be many benefits of making available, in an easily searchable form, the opinions and results of reviews made by thousands of ethics committees around the world. Some of these benefits include:

- Assistance to those seeking clarity or consensus about how to apply ethical principles in similar situations

- Fostering the detection of inconsistencies in use of terminologies

- Generating standardized forms, in a compatible way, across investigations

- Providing a standardized vocabulary for submissions to regulatory agencies

- Improving the collection of data from past studies ${ }^{1}$

- Increased efficiency, accuracy, and standardization.

For example, an ethics committee in one location that is reviewing a protocol involving drugs, devices, or groups of potential subjects that is similar to a study being conducted elsewhere, might benefit from the opinions and judgments about common ethical concerns, if only the two groups could share such information. While there are bulletins and peer-reviewed journals that discuss biomedical ethics deliberations, the details of such deliberations remain largely unused and difficult to search.

Even though there are electronic resources that support basic biomedical ethics searches such as the Thesaurus Ethics in the Life Sciences, ${ }^{2}$ there is as yet no standardized representation of the basic terms and entities existing in the biomedical domain of a sort that would support reasoning - and this is so even as ethics committees move towards keeping electronic records of their work. As with many legislative or governmental mandates, the standards for applying biomedical ethical concerns to human subject protocols are often poorly defined, or are difficult to apply to new situations. They also seem to have often been developed in ad hoc ways.

There are practical problems associated with accurately and efficiently collecting, classifying, and annotating the information that ethics committees use. One approach to this problem that has met with some success in other fields is the building of ontologies. Creating a Biomedical Ethics Ontology would make it possible to archive the results of ethics committee deliberations, search that information intelligently, and put it to future use in a number of ways. It would also facilitate multi-region

Journal of Empirical Research on Human Research Ethics, PP. 43-58. PRINT ISSN 1556-2646, ONLINE ISSN 1556-2654. () 2009 BY JOAN SIEBER. ALL RIGHTS RESERVED. PLEASE DIRECT ALL REQUESTS FOR PERMISSIONS TO PHOTOCOPY OR REPRODUCE ARTICLE CONTENT THROUGH THE UNIVERSITY OF CALIFORNIA PRESS'S RIGHTS AND PERMISSIONS WEBSITE, HTTP://WWW.UCPRESSJOURNALS.COM/REPRINTINFO.ASP. DOI: $10.1525 /$ jer.2009.4.1.43 

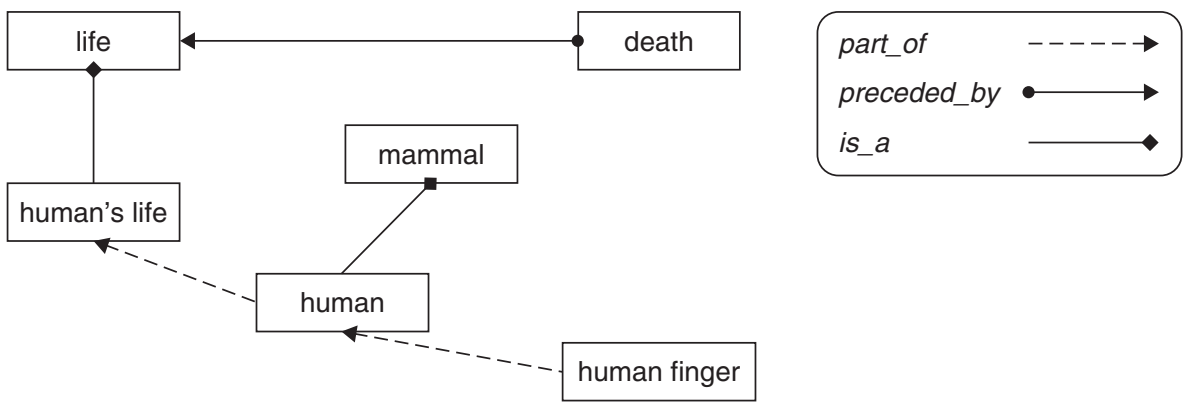

FIG. 1. A Highly Simplistic Domain Ontology.

and international protocol reviews where differing regulations and standards impose logistical, legal, and ethical complexities.

First, we will look at the science behind ontologies. Then, we will discuss how ontologies can be applied to the problems of human subjects research, and eventually be put into use by expert systems and IRB members.

\section{Ontologies}

Humans are natural sorters. We categorize all kinds of things, ranging from ideas, and arguments, to molecules, and planets. One way to categorize things is by sorting them into domains. A domain is an aspect of reality that humans seek to know, understand, and explain (also, predict, manipulate, and control) as fully as is possible through the development of a field of knowledge. Examples include all of the various subjects investigated at a typical university.

Since the rise of the computer age and the use of the World Wide Web for the dissemination of data and information, informaticians in various domains have begun to use computers to categorize their information into domain ontologies. A domain ontology is a structured representation of the entities in a specific domain such as geography, ecology, law, genomics, or medicine, including representation of important relations among elements (Smith, 2003). Entities are labeled with terms such as human, human finger, human's life, mammal, life, and death, while the relations get labels such as is_a, part_of, preceded_by, and others (Figure 1). In computertractable language, we might write:

- Human is_a mammal (meaning "human is a subtype of mammal").

- Human finger part_of human (meaning "human finger is a part of human").

- Human participant_in human's life (meaning "human is a participant in [the process which is a] human's life").
- Death preceded_by life (meaning "death is preceded by life").

The data and information from domain ontologies (that is, terms representing entities and relations) can be entered into a computer so that they may be made available and reasoned with by software, and may also be put together to form tree-like graphs, as shown in Figures 2 and 3. These tools help both computers and humans use complex information, and understand relations among critical terms and concepts.

A domain ontology usually evolves in several steps. First, the terminology used in the ontology should be laid down in a lexicon. A lexicon is a structured vocabulary of terms and their Aristotelian definitions (Jansen, 2008; Harari, 2004; Pelligrin, 1987), and its key benefit is

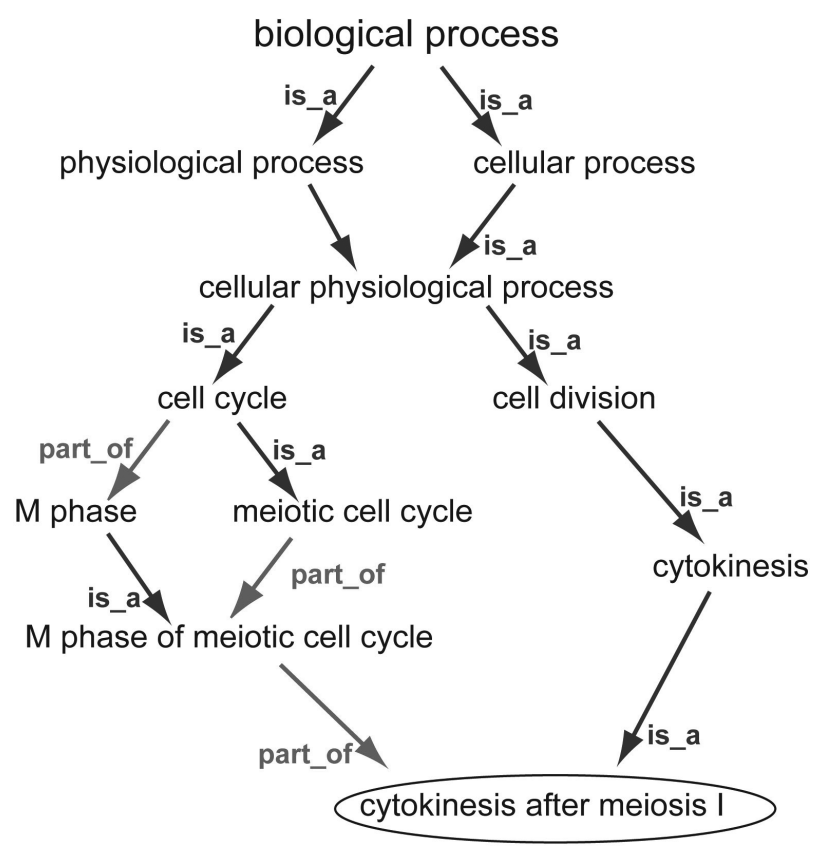

FIG. 2. Part of a Cell Domain Ontology. 


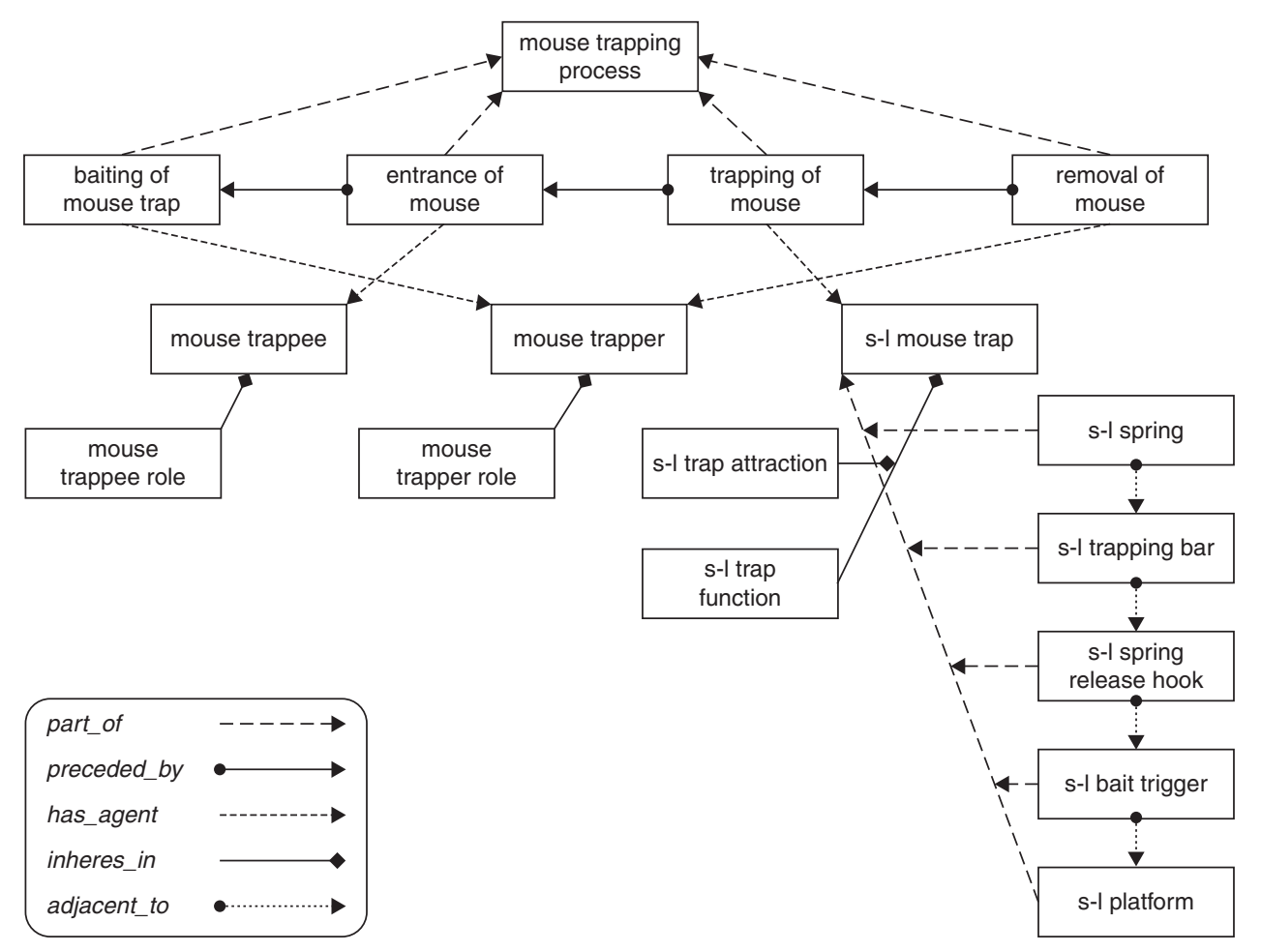

FIG. 3. The Beginnings of a Mouse Trap Annotation with Ontological Terms.

better communication through shared language. The definitions in a good lexicon will contain many instances of the phrase is_a, and the Aristotelian form "A is_a $\mathrm{B}$ that has C" or "A is_a B that Cs." So, for example:

- A human (A) is an animal (B) that is rational (C).

- Bioethics (A) is a branch of philosophy (B) that studies bioethical decisions $(\mathrm{C})$.

- A mouse trap (A) is a rodent trap (B) that traps mice (C).

After clear and coherent definitions of entities are developed, then all of the relations among those entities must be classified and defined. Then, the entities and relations of the domain ontology must be linked to one another, keeping in mind any axiomatic rules that guide their formation. As an example of an axiomatic rule, consider the natural axiom of transitivity that is present in any standard taxonomy containing the is_a "is a subtype of" relation: if $\mathrm{X}$ is_a $\mathrm{Y}$, and $\mathrm{Y}$ is_a $\mathrm{Z}$, then $\mathrm{X}$ is_a $\mathrm{Z}$. In the Linnean Cladistic taxonomy that forms the basis for the classification of biological kingdoms, we can see that:

- Homo sapiens is_a Homo, and Homo is_a hominid, therefore Homo sapiens is_a hominid.

- Chordate is_a animal, and animal is_a eukaryote, therefore chordate is_a eukaryote.
Ideally, a domain ontology is a standardized taxonomic classification that provides a controlled, structured vocabulary and framework to annotate data within a particular domain, in order to make the data more easily searchable by human beings and processable by computers. The following is a list of the several empirically verified benefits gained from utilizing an ontology (also see Smith et al., 2007; Arp et al., 2008):

- Formation of a controlled vocabulary of terms

- Development of clearly defined terms and relations between terms in a structured taxonomy

- Assistance in the process of normalization of terms used among practitioners

- Fostering interoperability of databases

- Support for computation reasoning on comprehensive bodies of collected data

- Improvement of searches-e.g., you can find child terms (items that are members of subclasses of other classes in a taxonomy) by using the transitive is_a reasoning mentioned above.

Biomedical ontologies exist and are being created for a variety of applications. They are useful in developing computerized diagnostic agents and in automating electronic health records and databases. By adding a 
semantic layer (a layer that includes meaning) to large collections of information, studies can be conducted on old databases, and new information can be better integrated and reasoned with for emerging needs. Ultimately, by standardizing vocabularies and defining relations among known objects in the biomedical domain, communication, and reasoning among different medical disciplines will be facilitated.

Current projects in biomedical ontology are at varying stages of development. In the health care field, ontologies are an important part of developing interoperable health information technology as medical communities move toward adopting electronic health record databases. In biology, ontologies have become essential for annotating the literature and integrating multiple, diverse knowledge bases derived from numerous highdata-yield experiments and research programs (Smith et al., 2007; Rubin et al., 2006). Examples include the Gene Ontology, the Foundational Model of Anatomy, the Cell Ontology, the Protein Ontology, the Disease Ontology, and many others that are found in Open Biomedical Ontologies Foundry. ${ }^{3}$ As a result of these projects, members of related disciplines, as well as members of single disciplines working on different subspecialties, or at different locations, are now able to communicate more clearly, share more information, and collaborate more readily. They are also able to search and sort within large repositories of information with greater precision.

\section{The Biomedical Ethics Domain}

Biomedical investigations are typically complex processes, involving numerous parties frequently in geographically distinct areas, and often taking place over lengthy spans of time. Drugs, devices, and procedures intended to treat or cure disabilities, diseases, or defects must be tested under a variety of conditions. Eventually, human subjects may be used, and there may be significant risks involved in the clinical trial. Because of lapses in scientific and ethical judgments by researchers, human subjects have sometimes suffered unnecessarily (Childress, Meslin, \& Shapiro, 2005; Murphy, 2004).

The principles of biomedical ethics that are intended to guide decision making in human subjects research are relatively settled, even though individual reviewers might differ in applying them to specific protocols. The mandated requirements for reviewing protocols and consent forms are also well settled. ${ }^{4}$ In practice, applying these requirements and principles often results in a divergence of opinions, and new cases pose challenges that can require lengthy debate, consultations with researchers and other experts, and produce varying decisions. All of this can be costly and time-intensive, delaying the start of human trials, and requiring reams of paperwork. A typical packet for an ethics committee member might consist of hundreds of pages of documents detailing protocols, proposed consent forms, and other standardized forms that have been filled in by researchers. Finally, each committee member must make recommendations for protocols by filling in standardized forms capturing their impressions of the rigor and ethics of a particular proposed study. All of these data could be extraordinarily useful to other ethics committees, and could help reduce costs, time, and puzzlement by disparate groups of researchers and ethics boards confronted with similar situations. Moreover, if captured with reference to specific cultural contexts, it could foster awareness of the impact of cultural and other contextual factors in ethical decision making. This could enable further useful forensic studies of connections of particular ethical norms to those cultural contexts. Unfortunately, currently, much of this information goes largely uncaptured (or at least, un-usefully preserved), and some significant potential benefits of migrating to electronic records in the review process may be missed entirely because of the absence of a sound, standardized vocabulary underlying the effort.

Developing a standardized taxonomic classification (an ontology) that could define, organize, and track entities and processes in the biomedical ethics domain will help ethics committees in a number of ways. As new cases are added to a searchable database of real-world research ethics problems and their solutions, using a standardized taxonomic classification, committee members, clinicians, and researchers from around the world will be able to understand and use the descriptions of these cases in a common way and thus be better able to communicate about them. Analyzing issues in disparate research groups would be facilitated, and a better basis for future decision making will be built. An ethics committee considering a new protocol and consent documents could check the database, and find similar instances with greater ease. As the database grows, and the ontology is refined, decisions about new cases will be aided by comparing them with old cases based on wellestablished principles of biomedical ethics. Obviously, new cases may raise new ethical considerations; the ontology will facilitate recognition of both distinctions and similarities, thus building a more nuanced understanding of how ethical principles are applied in relation to changing contexts. Ultimately, an ontology that links together common elements of electronic ethics committee records will enable committee members to focus more 
on philosophical issues such as duty, care, and compassion, and require them to spend less time on those elements of the review process that can be automated through a robust ontology. Such an ontology will never replace ethical decision making by committees and individuals interacting in clinical and research settings, but it certainly would augment and streamline various elements of typical processes.

An Ontology for Biomedical Investigations (OBI) is already being developed, along with an Ontology for Clinical Investigations (OCI), ${ }^{5}$ so it would be a natural and timely addition to begin to develop a Biomedical Ethics Ontology (BMEO) for use in the ethics review process. The OBI lacks focus, however, on specifically ethical and regulatory issues involved in ethics committee reviews, and focuses rather on investigational procedures, protocols, and methodologies. A BMEO will fill in a gap, and provide benefits specifically to ethics committees. How can this vision be achieved? Next we look briefly at the notion of biomedical ethics and its principles, and then consider how we might devise a controlled vocabulary for ontological uses.

\section{Method}

A BMEO would include rigorous definitions of biomedical ethics terms. For instance, not only should terms like autonomy be well defined, but given the vast repository of cases in the literature, examples of autonomy and counter-examples can be compared. The same should be done with the terms informed consent, minimal risk, harm, study, research, human subject, sample, vulnerable population, and other similarly critical terms. The goal should be to correlate definitions based not just on first principles, but in conjunction with canonical (textbook) cases, with expert medical and philosophical definitions of these terms. Because the field is an applied one, pure theory can be matched with cases to develop instances matching the types, and good correlates must be found for canonical instances for each core term.

Core terms, such as beneficence, autonomy, risk, justice, vulnerable population, informed consent, nonmaleficence, and others, must be mined from the central institutional documents for directing ethical treatment for human subjects in research. Examples of some repositories of core terms include the Council for International Organizations of Medical Sciences (CIOMS) and the "International Ethical Guidelines for Biomedical Research Involving Human Subjects." As an example of how these documents and terms may relate, the term minimal risk central to the IRB process in the U.S. is mentioned in the CIOMS document, but is absent from the EU Directive (see http://whqlibdoc. who.int/emro/2004/9290213639_annex2.pdf). While minimal risk research is afforded "expedited review" under the U.S. 45 C.F.R. 46 , without a similar provision in cooperating institutions involved in multi-national, multi-center studies, differing review standards and processes would seem to complicate these sorts of studies. The BMEO would highlight such differences and provide a bridge between critical similarities. This should help ease the design and implementation of international human subjects research protocols.

Are there similar terms, or related core concepts that need to be standardized, and can doing so streamline review procedures, as well as ensure ethical treatment of human subjects? A thorough study of all the relevant documents, institutions, and their procedures should reveal other examples, and pave a path toward a standardized taxonomic classification-a BMEO-that can be implemented on the World Wide Web and will assist in defining, organizing, and tracking the various entities in the biomedical ethics domain. Moreover, as the definitions and usages of terms change over time, a centralized ontology-maintained by specialists in the community of researchers and cooperating ontologistswill ensure that the latest definitions and working usages of common terms are propagated throughout the IRB community as a whole as rapidly as possible.

\section{Examples and Problems}

A typical ethics review begins with the dissemination among the committee members of the study packet along with various standardized forms filled out by the investigators. Even at this point, in the generation of standardized forms, the BMEO would aid both researchers and review committees.

For instance, consent forms involve legal and ethical requirements for providing informed consent to human subjects. Hundreds of thousands of individualized consent forms have been generated since such consent was first required. Understanding the relations between particular types of subjects in known contexts, and disclosures necessary for fully informed consent, is an ontological problem. Consent is informed if, under certain known conditions, certain types of subjects are notified in a particular manner. By generating an ontology of these relations, classifying types of subjects, contexts, and disclosures, much of the generation of consent forms could be made easier and more accurate. The information that will be used for generating this ontology lies in each new approved consent from all past studies (with the most recent studies begin most relevant) 
and that data can and should be mined to help develop an ontology of informed consent. Such an ontology can sit behind the scenes in software that will help researchers generate consent forms by answering various queries about the types of subjects, contexts, procedures, and protocols involved in a study. The consent forms generated will still require some editing by the investigators, but much of the language involved in these consents is more or less "boiler-plate" that has generally been tried and tested in previous studies. Relying on a querying mechanism through specialized software, software utilizing the BMEO will reduce the sometimes unfortunate effects of "cutting and pasting" that currently occurs with proposed consent forms, and will invoke the repository of past knowledge currently lying dormant in reams of past consent documents and ethics committee decisions.

Now we can consider the value of the BMEO to studies being reviewed by various ethics committees. As noted by many, the terms and principles of theoretical bioethics, as applied through the rules and regulations of institutions devised to protect human subjects, remain problematically vague. According to Lehman and Rodgers (2001), one of the early threshold parts of the review process is plagued by vagueness. That step involves determining whether a study is exempt or whether it should be expedited. "The qualifications for exempt studies and expedited review and the requirements for informed consent are not clearly delineated owing to vague terminology" (p. 188). Studies may qualify for expedited review only in case they pose minimal risk to subjects, and minimal risk seems to be clearly defined by the regulations as "the probability and magnitude of harm or discomfort anticipated in research [being] not greater in and of themselves than those ordinarily encountered in daily life or during performance of routine physical or psychological examinations or tests" (U.S. 45 CFR Part 46; Lehman, 2001, pp. 190-191). A study may be expedited if it involves minimal risk, and falls under one of several specifically delineated categories of study. All of these various determinations are essentially ontological problems.

Consider a study involving adult subjects, not belonging to any vulnerable population, who will be administered pin-prick blood tests and a mouth swab for DNA testing, and are asked to run on a treadmill for an hour. If forms required to be filled out by researchers capture the essential categorical information about such a proposed study, then software running on top of a BMEO that reflects the essential qualities of exempt and expedited studies will automatically sort clear cases like this one. Moreover, this sorting can take account of individual locations and variations in ethical norms, rules, or regulations according to the geographical location of particular centers. Of course, numerous cases and exceptions will need to be identified to make such an ontology work. Again, ideally forms used by researchers will collect data on the subjects (e.g., Is the subject a hemophiliac? Then pin-pricks won't qualify for minimal risk) and either automatically sort the studies into expedited, exempt, or other categories, or suggest proper categories to the ethics committee based upon these forms. The forms currently used in most reviews, filled out by researchers, and typically reviewed by all members of the committee can serve as the template for the BMEO, which will then help automate at least the easy threshold sorting that goes on in the review process, leaving more time for committee members to focus on ethical inquiries that cannot be automated. This is already the case in medical ontologies, and is a large part of the value offered by both public and private ventures developing products based upon ontologies. ${ }^{6}$

So our hypothetical researcher of the future will be aided in putting together his or her study proposal for review, with the BMEO working in the background to help devise appropriate language for consent documents, and automatically suggesting whether, based on specified inputs to various pre-determined queries, the study should be sorted into an expedited or exempt track. Ethics committees will be aided by having those categorical tasks that can be realistically automated taken off their to-do lists so they can better concentrate on the elements of the review that so far only human reviewers can accomplish. All of this requires a great deal of research and cooperation within the ethics committee community and those interested in devising a BMEO, as well as access to electronic ethics committee documents undergoing the review process, and collaboration in defining terms and devising ontologies. We have taken a first step in this direction, by mapping out some key, high-level terms and relations describing the ethics review process itself.

\section{Results: The Beginnings of a Biomedical Ethics Ontology (BMEO)}

Our research program described herein is a proof of concept of the problems and methods involved in developing a BMEO and applying it in the domain of human subjects research. It is also a call for collaboration on this effort from others in the field of research ethics and in related sciences.

In the previous section, we mentioned that a domain ontology is a taxonomy comprised of (a) entities and 


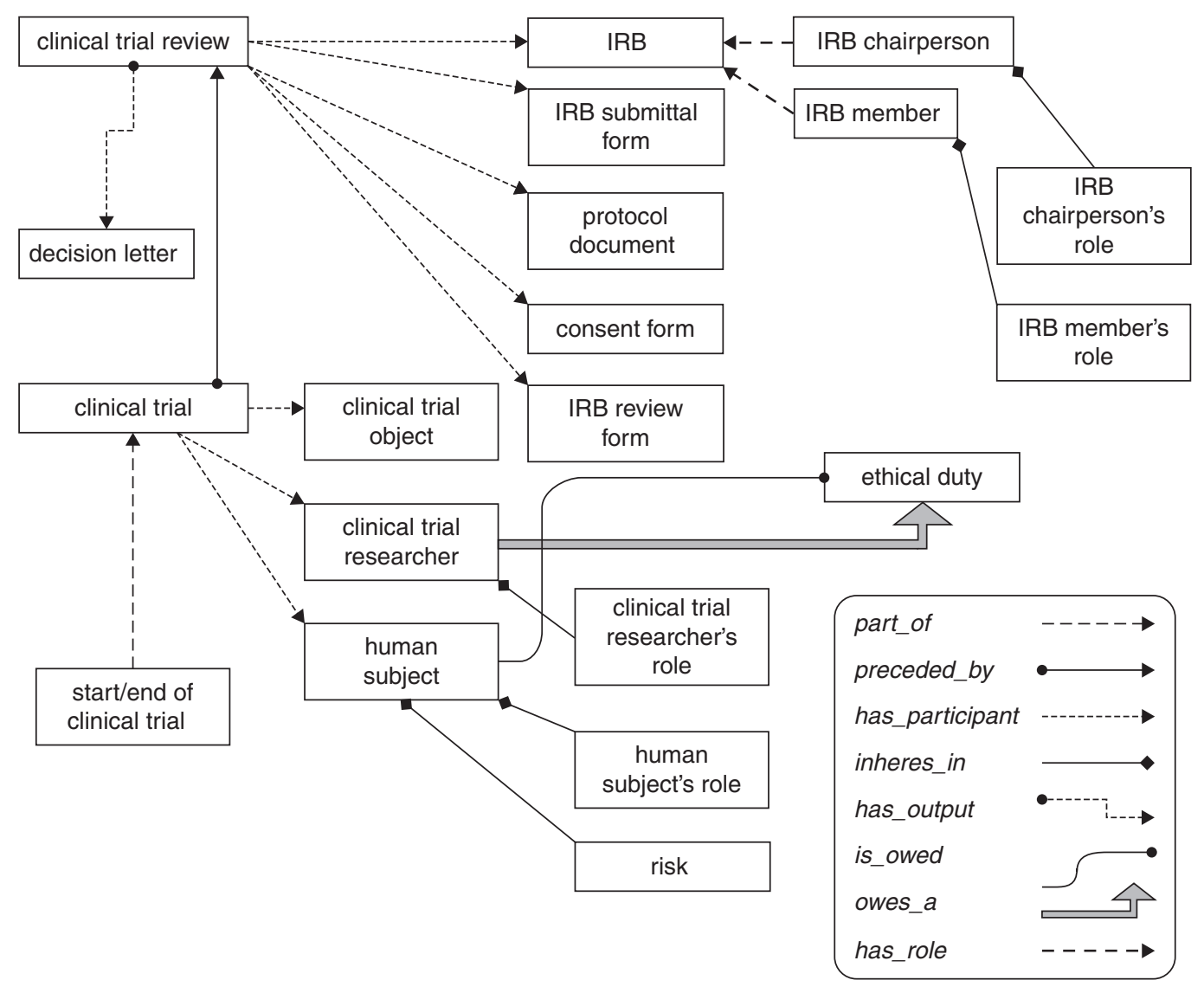

FIG. 4. The Beginnings of a BMEO with a Focus on IRBs.

(b) relations. These entities and relations are labeled with terms. We also mentioned that the first step in constructing a domain ontology involves defining the terms in a lexicon. Thus, we first generated a list of common entities associated with biomedical ethics in the IRB context and provided Aristotelian definitions-definitions of the form "A is_a B that has C" or "A is_a B that Cs"-for each. This is a standard best-practice when beginning to construct a domain ontology, looking first at the "low-hanging fruit" of commonly used terms and relations in a particular domain. Our initial results are depicted in Appendix I.

We next began the task of turning this taxonomy into a domain ontology. The domain ontology incorporates other relationships beyond is_a, and adds one specifically applicable to ethical issues: owes_a for ethical duties. We use standard, common-sense relations used by thousands of researchers that can be found in the Open Biomedical Ontologies (OBO) Relation Ontology. ${ }^{7}$ Appendix II shows some of those relations that can be used for reasoning, which is one central goal of a welldescribed ontology. We have illustrated a few of these entities and relations in Figure 4.

\section{Discussion: Putting the BMEO to Use-Strategies and Roles}

Ontologies are built to:

- Be used

- Extend vocabularies among disparate sciences and specialists

- Develop tools for reasoning

- Automate tedious processes

- Be expanded and adjusted over time.

The above relations and definitions are being imported into an OWL (Web Ontology Language) file using Protégé, which is a free, open-source ontology editor and knowledge-base framework. ${ }^{8}$ As with other working ontologies, we will maintain a "wiki" and seek further input and contributions to extend and correct it over time. ${ }^{9}$ In order to be maximally useful, ontologies should be open source (accessible and editable to all interested and affected parties), and welcome ongoing comment from both domain experts and professional 
ontologists. We have described only a very small snapshot of a standard ethics committee review process and relations among its objects. Much more must be done before use can be made of what we are building, and contributions from others examining the domain will no doubt be necessary.

Recent history has shown that when ontologies are developed using as wide of a community as possible, engaged in a centralized and coordinated process, costly errors and duplicative efforts can be avoided. It will require cooperation among those interested in ensuring that electronic ethics committee records and processes are put to the best use as they evolve, and made most efficient. There will be little-to-no learning curve involved for users, or typical ethics committee members. Some domain specialists (e.g., ethics committee members, ethicists, and lawyers) will need to work with professional ontologists to construct a working ontology that then can be integrated into software. Empirical studies will need to be conducted to determine the accuracy and usefulness of ontologies as they develop. To realize the BMEO, we will first need to gather a group of ontologists and domain specialists sufficient to generate a useful number of terms and relations, and to begin putting it to real-world tests. Once empirical studies demonstrate the usefulness of a BMEO, through working software products, the domain ontology will still require maintenance and adjustment over time, but typical ethics committee members and coordinators will only need to involve themselves with ontology development and maintenance to the degree to which it interests them. Open-source domain ontologies are open to constant revision in light of new evidence, and other working ontologies evolve over time through voluntary editing by interested users and ontologists. Let us consider some of the potential uses that a future, more robust version of the BMEO will have.

The concepts of biomedical ethics are well-established through the literature and seminal documents already discussed, and the fact of the existence of various duties is widely accepted. However, the extent of those duties, and application of those principles into a coherent set of actions and intentions for every conceivable circumstance in clinical and research contexts is impossible to conceive. We will do best to not simply note instances or specific cases, but to describe in logical detail the principles underlying those cases. Thus, when we are describing a "duty of care," whether it be between a physician and patient, parent or child, or child and elder, the central principle "duty" is well-defined and understood across all related disciplines, and "curated," as it were, in the ontology. As a result, even as new needs require us to define new duties, the core principle remains an anchor for future and emerging discussions and eventually, norms. This is necessary because new biomedical technologies offer an ever-increasing spectrum of potential situations, each of which require analysis, debate, and consideration by ethics committees, researchers, and physicians. No machine running on top of any ontology, no matter how well conceived, can replace this process. But there are certainly ways in which a well-built ontology can streamline certain processes, and help gather useful information for the various interested parties to mine in beneficial ways.

With a stack of protocols to review, an ethics committee member typically is asked to be primary or secondary reviewer for a particular protocol. Their reviews are conducted according to established standards, and a typical review sheet might look like the one in Figure 5, aside from more detailed comments that might be included.

As discussed above, it seems clear that elements of forms like this could be easily automated by being developed electronically on top of a good ontology. Thus, for instance, checking the box for III.A (this study carries only a minimal risk) would automatically exclude other mutually exclusive options (represented logically in the OWL file for the BMEO by a disjoint between "minimal risk" and "nonminimal risk"). The class of procedures involving a minimal risk is well defined, and is represented in the ontology. A database built on the BMEO ontology could collect cases, or instances, and associate them with these classes. Ethics committee members, faced with new protocols for which they may have doubts about the risk associated with certain procedures, could search those instances, or an artificial agent could suggest sorting a study into either more-than-minimal risk or minimal risk based upon the archive of instances, rather than requiring individual committee members to determine this threshold, except perhaps in difficult cases which would be flagged by such an agent where there is a dearth of similar cases.

A fair amount of ethics committee time and energy goes into determining the adequacy of the "consent" documents. A well-built ontology could help at the front end of the process by providing a way for researchers submitting protocols to put together well-formed consent documents based upon a repository of adequately phrased consents already approved in the past. Moreover, the ontology could help track the extent and coverage of particular portions of the consent documents for future uses of data acquired through a particular investigation. The ontology could help coordinate and track elements of a consent document and their associations with different phases of a study. Thus, a particular sample would always carry with it the particular part of the 


\begin{tabular}{|c|c|c|c|}
\hline I. Research Design & Yes & No & NA \\
\hline A. Rationale & $x$ & & \\
\hline B. Objective/Problem Statement & $x$ & & \\
\hline C. Background, Previous Studies & $x$ & & \\
\hline D. Methods & $x$ & & \\
\hline 1. Experimental vs. standard & $x$ & & \\
\hline \multicolumn{4}{|l|}{ 2a. Required for standard care } \\
\hline \multirow{2}{*}{\multicolumn{4}{|c|}{$2 b$. Required only for purpose of research }} \\
\hline 3. Use of placebo (if applicable) & & & \\
\hline 4. Likelihood of accomplishing objective & $x$ & & \\
\hline E. Date Safety Monitoring Plan & $x$ & & \\
\hline F. Statistical Plan & $\mathrm{X}$ & & \\
\hline G. Mechanism for Reporting SAEs (serious adverse events) & $x$ & & \\
\hline III. Risks/Benefits & Yes & No & NA \\
\hline A. Minimal Risk & & $x$ & \\
\hline B. Greater than Minimal Risk but presenting the prospect of direct benefit & $\mathrm{x}$ & & \\
\hline \multicolumn{4}{|l|}{$\begin{array}{l}\text { C. Greater than Minimal Risk and no prospect of direct benefit but likely } \\
\text { to yield generalizable knowledge }\end{array}$} \\
\hline D. Protections in place for vulnerable populations & & & $X$ \\
\hline \multicolumn{4}{|l|}{ IV. Consent Document/Assent Document Includes the Following Required } \\
\hline Elements (additional comments can be made under Part V): & Yes & No & Inadequate \\
\hline $\begin{array}{l}\text { 1. A statement the study involves research, an explanation of the purposes of the research, } \\
\text { the expected duration of the subject's participation, a description of the procedures } \\
\text { to be followed, and identification of any procedures that are experimental. }\end{array}$ & $\mathrm{x}$ & & \\
\hline 2. A description of any reasonably foreseeable risks and discomforts to the subject. & $x$ & & \\
\hline $\begin{array}{l}\text { 3. A description of any benefits to the subject or to others which may reasonably be expected } \\
\text { from the research. }\end{array}$ & $x$ & & \\
\hline $\begin{array}{l}\text { 4. A disclosure of appropriate alternative procedures or courses of treatment, if any, that might } \\
\text { be advantageous to the subject. }\end{array}$ & $\mathrm{X}$ & & \\
\hline $\begin{array}{l}\text { 5. A statement describing the extent, if any, to which confidentiality of records identifying the } \\
\text { subject will be maintained and that notes the possibility that the U.S. Food and Drug } \\
\text { Administration and/or the pharmaceutical company may inspect the records. }\end{array}$ & $x$ & & \\
\hline $\begin{array}{l}\text { 6. For research involving more than minimal risk, an explanation as to whether any compensation } \\
\text { and an explanation as to whether anymedical treatments are available if injury does occur and, } \\
\text { if so, what they consist of, or where further information may be obtained. }\end{array}$ & $x$ & & \\
\hline $\begin{array}{l}\text { 7. An explanation of whom to contact for answers to pertinent questions about the research and research } \\
\text { subjects' rights, and whom to contact in the event of a research-related injury to the subject. }\end{array}$ & $x$ & & \\
\hline $\begin{array}{l}\text { 8. A statement that participation is voluntary, that refusal to participate will involve no penalty } \\
\text { or loss of benefits to which the subject is otherwise entitled, and that the subject may } \\
\text { discontinue participation at any time without penalty or loss of benefits to which the subject } \\
\text { is otherwise entitled. }\end{array}$ & $x$ & & \\
\hline \multicolumn{4}{|l|}{ Principal Investigator: } \\
\hline \multicolumn{4}{|l|}{ Optional Elements of Informed Consent } \\
\hline \multicolumn{4}{|l|}{ When Appropriate, One or More of the Following Elements Shall Also be } \\
\hline $\begin{array}{l}\text { 1. A statement that the particular treatment or procedure may involve risks to the subject (or to the } \\
\text { embryo or fetus, if the subject is or may become pregnant), which are currently unforeseeable. }\end{array}$ & $\mathrm{x}$ & & \\
\hline $\begin{array}{l}\text { 2. Anticipated circumstances under which the subject's participation may be terminated } \\
\text { by the investigator without regard to the subject's consent. }\end{array}$ & $x$ & & \\
\hline 3. Any additional costs to the subject that may result from participation in the research. & $x$ & & \\
\hline $\begin{array}{l}\text { 4. The consequences of a subject's decision to withdraw from the research and procedures } \\
\text { for orderly termination of participation by the subject. }\end{array}$ & $x$ & & \\
\hline $\begin{array}{l}\text { 5. A statement that significant new findings developed during the course of the research that may } \\
\text { relate to the subject's willingness to continue participation will be provided to the subject. }\end{array}$ & $\mathrm{x}$ & & \\
\hline 6. The approximate number of subjects involved in the study. & $x$ & & \\
\hline
\end{tabular}

FIG. 5. Typical IRB Review Form. 
consent associated with it, and define the extent of its uses in the future. Databases that use the BMEO, cataloguing samples in biobanks, will help ensure that rights and obligations specifically gathered by particular consent documents, remain with each particular sample. All of these benefits should help ensure greater protection of human subjects, and greater efficiency for ethics committees.

\section{Research Agenda: Groundwork for Future Study}

A BMEO has use in clinical medicine as well as clinical trials and human subjects research in general. Ethics committees at hospitals and research institutions should be able to use an ontology of biomedical ethics terms to standardize their vocabularies and training, as well as link to repositories of cases indexed according to these terms to correlate new cases and their ethical judgments among one another. This is just one of the eventual uses of a Biomedical Ethics Ontology. As the work proceeds on mining existing documents for core ethical terms, and creating a vocabulary of standard procedures in the realm of human subjects protocols, case studies will be developed to test its use in clinical trials. We will test the ontology on a hypothetical multi-national, multi-center trial involving human subjects.

For the purpose of further case studies, more hypothetical trials will be devised, a protocol written, and the various institutional and governmental requirements for ethical human subject research from between three and five locales (with differing standards and institutions) will be used. We will use a prototype Biomedical Ethics Ontology to standardize review procedures and in filling out checklists for requirements in an electronic review form incorporating ethical and procedural requirements in protocol reviews across all the hypothetical study centers. We will test these hypotheticals against real studies and real review forms and associated comments that have been de-identified or otherwise useable without breaching confidentiality. A statistically significant number of real-world cases will be used and tested against idealized types.

We expect that this process will enable the creation of electronic protocol review forms and consent documents that can automate those elements of reviews requiring little human judgment. Also, we should expect that human reviewers will be aided not only by streamlining elements of the review that can be automated, but by calling attention to differences in various review center procedures, standards, and laws. Local ethics committees will be better equipped to understand local rules, and to calibrate study designs in the future to meet both common and uncommon requirements and procedures as multi-national studies become more commonplace. At the end of the five-year study, we expect we will have completed a robust ontology of biomedical ethics, and proved its use in hypothetical cases, and will have devised automated procedures for streamlining ethical committee reviews in multi-center and international clinical trials. All of this will require a core set of dedicated domain specialists and ontologists, working together, using archival, hypothetical, and experimental data, properly de-identified, to devise a working ontology that can be incorporated into useful software.

\section{Best Practices}

The BMEO project, however it is ultimately funded and composed, must become affiliated with the existing OBO Foundry consortium to take full advantage of legacy data, best-practice expertise already accumulated, and efficiencies of scale. The OBO Foundry has an existing set of Best Practices principles that are guiding ontology development among its ongoing projects. Those principles are quoted here, and available on the World Wide Web for further commentary and refinement.

(Version as of 24 April 2006.) Further principles will be added over time.

1. The ontology is open and available to be used by all without any constraint other than (1) its origin must be acknowledged and (2) it is not to be altered and subsequently redistributed under the original name or with the same identifiers.

2. The ontology is in, or can be expressed in, a common formal language. A provisional list of languages supported by $\mathrm{OBO}$ is provided at http://obo.sf.net/.

3. The ontology possesses a unique identifier space within $\mathrm{OBO}$.

4. The ontology provider has procedures for identifying distinct successive versions.

5. The ontology has a clearly specified and clearly delineated content.

6. The ontology includes textual definitions for all terms.

7. The ontology uses relations which are unambiguously defined following the pattern of definitions laid down in the OBO Relation Ontology.

8. The ontology is well-documented.

9. The ontology has a plurality of independent users.

10. The ontologies in the OBO Foundry will be developed in a collaborative effort. ${ }^{10}$ 
The BMEO is a work in progress. We welcome comments and contributions from others similarly interested in realizing its promise. While we search for funding and develop test cases, the ontology itself will be publicly available for review, comment, and contribution at the OBO Foundry website and our wiki will also remain publicly available.

Successful ontology development faces numerous challenges in all domains, chiefly involving community participation and support. Once a core group of both domain specialists and ontologists agrees to pursue developing a BMEO, several years of study will be required, and a working prototype useful to the ethics committee community must be created. As with any tool, its value will be proportional to the usefulness of the product created. The project should involve as many interested and knowledgeable researchers as possible. This then is a call to all those interested to become involved as we pursue the creation, testing, and ultimately deployment of a technology that could dramatically assist in ethics review processes. We look forward to commentary and criticism of the proposed methodology as we pursue wider involvement, interest, and commitment for developing a BMEO.

\section{Acknowledgments}

This work was supported in part by the National Center for Ontological Research and the State University of New York at Buffalo. The contribution of Jennifer Fostel to this work was supported by the Division of Intramural Research of the National Institute of Environmental Health Science, under contract HHSN273200700046U. This work was funded by the National Institutes of Health through the NIH Roadmap for Medical Research, Grant 1 U 54 HG004028 (National Center for Biomedical Ontology).

\section{Author Note}

Address correspondence to: David Koepsell, Department of Philosophy, Delft University of Technology, Faculty of Technology, Policy and Management, P. O. Box 5015, 2600 GA Delft, The Netherlands. Phone (716) 913-2422; E-MAIL: drkoepsell@yahoo.com.

\section{Authors' Biographical Sketches}

David Koepsell has a law degree and Ph.D. in Philosophy from the University at Buffalo. He has authored numerous scholarly and popular articles, and has authored and edited several books in the fields of ethics, ontology, and legal philosophy. He is Assistant Professor, Section of Philosophy, Department of Technology, Policy, and Management at the Delft University of Technology, The Netherlands. He is the author of The Ontology of Cyberspace: Law, Philosophy and the Future of Intellectual Property (Open Court, 2000). His book, Who Owns You? The Corporate Gold-Rush to Patent Your Genes, was published by Wiley-Blackwell in 2009. He has served on the health sciences IRB at the University of Buffalo.

Robert Arp has a Ph.D. in Philosophy from Saint Louis University (Missouri) and is a Research Associate through the National Center for Biomedical Ontology at SUNY-Buffalo. Specializing in Biomedical Ontology and Philosophy of Biology, he has authored numerous philosophical and scientific articles, and is writing and editing several books in these fields. His book, Scenario Visualization: An Evolutionary Account of Creative Problem Solving, was published by MIT Press in 2008.

Jennifer M. Fostel supports the development of the Chemical Effects in Biological Systems (CEBS) Knowledgebase at the National Institute of Environmental Health Science (NIEHS) in Research Triangle Park, North Carolina, through a contract with SRA International. She is active in several public efforts to standardize and exchange data, including the Ontology for Biomedical Investigations (OBI) Working Group, the Clinical Data Interchange Standards Consortium (CDISC)/Standards for Exchange of Nonclinical Data (SEND), and sits on the Advisory Board for the MGED Society. Before joining the NIEHS CEBS effort, she directed research in mechanistic toxicology and antiinfectives discovery research at Abbott Laboratories (Abbott Park, Illinois) and Pharmacia Corporation (Kalamazoo, Michigan). During her work in industry, she developed methods to analyze, annotate and integrate -omics data from transcriptomics, proteomics, and metabonomics in various model organisms

Barry Smith is Julian Park Distinguished Professor of Philosophy at the University at Buffalo (New York) and Research Scientist in the New York State Center of Excellence in Bioinformatics and Life Sciences. He is the author of some 450 scientific publications, including 15 authored or edited books, and his research has been funded by the National Institutes of Health; the U.S., Swiss, and Austrian National Science Foundations; the Volkswagen Foundation; and the European Union. He is a coordinating editor of the OBO (Open Biomedical Ontologies) Foundry and a member of the Scientific Advisory Board of the Gene Ontology Consortium, the OBI (Ontology for Biomedical Investigations) Working Group, and of the Cleveland Clinic Semantic Database for Cardiovascular Surgery. 
Author Contributions. David Koepsell co-conceived of the project, drafted, edited, and researched the topic, and provided expertise in biomedical ethics and ethics committee procedures. Robert Arp drafted and edited, devised drafts of the ontology, and provided expertise in biomedical ethics. Jennifer Fostel reviewed and edited the draft paper, revised the ontology and appendices, and provided expertise in clinical investigations and biomedical ontologies. Barry Smith co-conceived of the project, provided research and support, edited and revised the ontology and appendices, and provided expertise in ontology development and biomedical ontologies.

\section{End Notes}

${ }^{1}$ In fact, analogues of these benefits are already beginning to be manifested through the Ontology for Biomedical Investigations (OBI) project. OBI is developing an integrated ontology for the description of biological and clinical investigations; see http://obi-ontology.org/page/ Main_Page.
${ }^{2}$ The German Reference Centre for Ethics in the Life Sciences (DRZE) is affiliated with the University of Bonn and is concerned with the "prerequisites, norms and criteria of ethical judgment formation in the life sciences" (http://www.drze.de/dasdrze). The DRZE has produced what has come to be known as the "Bonn Thesaurus," a Thesaurus Ethics in the Life Sciences, now in its fifth edition; see http://www.drze.de/BELIT/thesaurus/.

${ }^{3}$ See http://www.obofoundry.org.

${ }^{4}$ See, for example, http://www.hhs.gov/ohrp/humansubjects/guidance/belmont.htm, http://www.hhs.gov/ ohrp/about/ohrpfactsheet.htm, and Beauchamp and Childress (2008).

${ }^{5} \mathrm{OBI}$ : http://obi.sourceforge.net/, OCI: http://www. bioontology.org/wiki/index.php/OCI:Main_Page.

${ }^{6}$ See, e.g., http://www.ontologyworks.com.

${ }^{7}$ See http://www.obofoundry.org/ro/.

${ }^{8}$ See http://protege.stanford.edu/.

${ }^{9}$ See http://libweb.lib.buffalo.edu/dokuwiki/hslwiki/ doku.php?id=bioethics_ontology.

${ }^{10} \mathrm{See} \mathrm{http} / / /$ wings.buffalo.edu/faculty/research/obofoundry/.

\section{References}

Arp, R., Romagnoli, C., Chhem, R., \& Overton, J. (2008). Radiological and biomedical knowledge integration: The ontological way. In R. Chhem, K. Hibbert, \& T. Van Deven (Eds.), Radiology education (pp. 87-104). Berlin: SpringerVerlag.

Beauchamp, T. \& Childress, J. (2008). Principles of biomedical ethics, 5th edition. Oxford: Oxford University Press.

Childress, J., Meslin, E., \& Shapiro, H. (Eds.) (2005). Belmont revisited: Ethical principles for research with human subjects. Washington, D.C.: Georgetown University Press.

Englehardt, T. (ED.) (2006). Global bioethics: The collapse of consensus. Salem, MA: M \& M Scrivener Press.

Harari, O. (2004). Knowledge and demonstration: Aristotle's posterior analytics. Dordrecht: Kluwer Academic Publishers.

Iltis, A., Johnson, S., \& Hinze, B. (Eds.) (2007). Legal perspectives in bioethics. London: Routledge.

Jansen, L. (2008). Categories: The top-level ontology. In K. Munn \& B. Smith (Eds.), Applied ontology: An introduction (pp. 173-196). Amsterdam: IOS Press.

Jonsen, A. (1998). The birth of bioethics. Oxford: Oxford University Press.

Lehman, C. M. \& Rodgers, G. M.(2001). To IRB or not to IRB? American Journal of Clinical Pathology, 115(2), 187-191.

Murphy, T. (2004). Case studies in biomedical research ethics. Cambridge: MIT Press.

Nirenberg, S. \& Raskin, V. (2004). Ontological semantics. Cambridge: MIT Press.
Pellegrin, P. (1987). Aristotle's classification of animals: Biology and conceptual unity of the Aristotelian corpus. Berkeley: University of California Press.

Percival, T. (1803/1849). Percival's medical ethics. London: John Churchill Publishers.

Rubin, D. L., Lewis, S. E., Mungall, C. J., Misra, S., Westerfield, M., Ashburner, M., Sim, I., Chute, C. G., Solbrig, H., Storey, M.-A., Smith, B., DayRichter, J., Noy, N. F., Musen, M. A. (2006). National Center for Biomedical Ontology: Advancing biomedicine through structured organization of scientific knowledge. Omics: A Journal of Integrative Biology, 10(2), 185-198.

Smith, B. (2003). Ontology. In L. Floridi (Ed.), Blackwell guide to the philosophy of computing and information (pp. 155-166). Malden, MA: Blackwell.

Smith, B., Ashburner, M., Rosse, C., Bard, J., Bug, W., Ceusters, W., Goldberg, L. J., Eibeck, K., Ireland, A., Mungall, C. J., the OBi Consortium, Leontis, N., Rocca-Serra, P., Ruttenberg, A., Sansone, S.-A., Scheuermann, R. H., Shah, N., Whetzel, P. L., \& LEwIS, S. (2007). The OBO Foundry: Coordinated evolution of ontologies to support biomedical data integration. Nature Biotechnology, 25(11), 1251-1255.

Tauber, A. I. (2005). Patient autonomy and the ethics of responsibility. Cambridge: MIT Press.

Tollefsen, C. (2007). Biomedical research and beyond: Expanding the ethics of inquiry. London: Routledge. 
APPENDIX I. Terms.

- Person: Homo sapiens (alternate term: person)

OBI DEF: A human being regarded as an individual

- OBI DEF: Personnel role is a role that inheres in a person or organization and is realized in a process of planning, overseeing, reviewing, reporting, and/or implementing a study.

- OBI DEF: Worker role is a personnel role played by a party who executes a component of the study plan.

- Researcher role Add to OBI: Researcher role is a worker role that inheres in a person and realized through a process of investigating and seeking to explain and predict some aspect of reality.

- Clinical trial researcher

BMEO: A clinical trial researcher is a researcher that conducts research using human subjects in a clinical trial. OBI DEF: Clinical trial research role is a researcher role realized in (concretization of some clinical study design).

- OBI DEF: Study participant is a role of entities that is realized through the implementation of a study design.

- OBI DEF: Study subject role inheres in an entity and is realized by the implementation of: (1) an observation design specifying how the entity is to be observed, and/or (2) an intervention design specifying the protocols applied to anything participating in the study, whether they are treated or acting as control.

- Human subject OBI DEF: A human subject role is a study subject role that inheres in a person.

- Animal subject OBI DEF: Animal subject role is a study subject role that inheres in an organism other than a person (probably good to skip this one-it is a bit fraught)

- Investigation OBI DEF: An investigation is a process with the objective to generate an information entity by planning protocol applications included in an overall study design, carrying them out and documenting the results. In addition, an investigation can include a subprocess of interpreting the data to draw conclusions.

- Clinical study design OBI DEF: Plan for the precise procedure to be followed in a clinical trial, including planned and actual timing of events, choice of control group, method of allocating treatments, blinding methods; assigns a subject to pass through one or more epochs in the course of a trial. Specific design elements, e.g., crossover, parallel; dose-escalation [modified from Pocock, Clinical Trials: A Practical Approach]

- Clinical trial BMEO: A clinical trial is a process aggregate where tests, experiments, and other evaluations are carried out on human subjects in order to assess the effects of a clinical trial drug or clinical trial device.

- Drug role OBI DEF: Drug role is a role assigned to an entity by a regulatory agency that is designed to diagnose, treat, or prevent an abnormal medical condition.

- Add to OBI: Test article role; definition to be determined but to include material entity and device and materials of biological origin; this would be a child of study participant role as it is defined in the study design.

- Clinical trial drug:

A clinical trial drug is a role of a drug whereby that drug is the subject of study in a clinical trial.

OBI DEF: Clinical trial drug role is a test article role that inheres in a material entity-bearing drug role.

- Device OBI DEF: A device is a material that is manufactured by an organization or person, designed with the intent to perform a specific function or functions.

- Clinical trial device: A clinical trial device is a role of a device whereby that device is the subject of study in a clinical trial. OBI DEF: Clinical trial device role is a test article role that inheres in a device.

- Review

BMEO: A review is a process in which persons participate that involves their understanding, assessing, and evaluating some proposed idea or project.

- Clinical trial review

BMEO: A clinical trial review is a review that is performed by an institutional review board to determine whether a proposed clinical trial meets the standards of ethical duty by evaluating protocol and consent documents of clinical trials and has an output in the form of a decision letter.

- Expedited clinical trial review

BMEO: An expedited clinical trial review is a clinical trial review that is conducted by the institutional review board (IRB) chairperson because the proposed clinical trial involves minimal risk.

- Full clinical trial review

BMEO: A full clinical trial review is a clinical trial review that is not expedited and follows all of the steps of the review process.

- Board: A board is a group of persons that participates in some review.

- OBI DEF: Regulatory role is a role realized by the act of regulating or the state of being regulated.

- $\mathrm{OBI}$ DEF: A regulator role is a regulatory role realized through the process of ensuring compliance with laws, regulations, and established rules. 
APPENDIX I. Continued.

- OBI DEF: Role of Institutional Review Board is a regulator role inhering in an organization and realized by the process of determining if a research project conforms to ethical principles and federal regulations that protect the rights and welfare of human research subjects.

- Institutional review board (IRB)

BMEO: An institutional review board is a board composed of peer reviewers that participates in a clinical trial review pursuant to institutional and legislative mandates.

- IRB member

BMEO: An IRB member is a role of a person whereby that person is part of an institutional review board.

- IRB chairperson

BMEO: An IRB chairperson is a role of a person whereby that person is part of an institutional review board who acts as the head of the board.

- Narrative object OBI DEF: A narrative object is an information content entity that is a set of propositions.

- Information content entity OBI DEF: An information content entity is an entity that is generically dependent on some artifact and stands in relation of aboutness to some entity.

- Protocol OBI DEF: A protocol is a plan that outlines a set of instructions to achieve a specific objective relevant to the biomedical domain and that can be realized as an assay or material transformation. The level of detail and quantitative information given in a protocol is sufficient to communicate it between domain experts, so that it can be assumed that different domain experts will be able to independently reproduce the process.

- Document (not in OBI or BMEO at the moment)

- Protocol document: A protocol document is a document that describes the objective, design, methodology, statistical considerations, and organization of a clinical trial.

- Consent form: A consent form is a document that a human subject reads, reviews, and signs giving permission to participate in a clinical trial performance.

- Institutional review board (IRB) review form: An IRB form is a form that an institutional review board uses to assess whether a proposed clinical trial meets the standards of ethical duty by evaluating protocol and consent documents of clinical trials involving human subjects.

- IRB submittal form: An IRB submittal form is a document that a clinical researcher submits to an institutional review board-along with a protocol document and consent form-indicating that the clinical researcher would like the institutional review board to undertake a clinical trial review.

- IRB decision letter: An IRB decision letter is a document that an institutional review board issues to inform the clinical trial researcher that the proposed clinical trial performance is either approved, denied, or requires modification.

- OBI DEF: Specified input role is a study participant role that is played by an entity and that is specified as output of the process.

- Output role: An output role is a role that is played by some object that results from some process.

- Duty: A duty is a principle that encourages or binds a person to the performance of some action or intention.

- Ethical duty: An ethical duty is a duty that involves the rights and privileges of persons, human beings, and other animals with complex nervous systems.

- Beneficence: Beneficence is an ethical duty that entails acting in the best interest of a person, human being, or other animal with a complex nervous system by protecting and defending rights, and actively promoting good outcomes.

- Non-maleficence: Non-maleficence is an ethical duty that entails not intentionally harming a person, human being, or other animal with a complex nervous system, as well as removing or preventing harms.

- Autonomy: Autonomy is an ethical duty that entails treating persons with respect by allowing them to make well-informed choices, telling the truth, respecting privacy, protecting confidentiality, and assisting when asked to help make decisions.

- Justice: Justice is an ethical duty that entails treating persons with fairness relative to their situation in life, including meting out goods, services, and the like, according to need, contribution, or merit.

- Ethical duty performance: An ethical duty performance is a process that entails acting in accordance with an ethical duty.

- Risk: A risk is a disposition that inheres in a subject whereby the subject is susceptible to physical or mental harm.

- Minimal risk: A minimal risk is a risk whereby the probability and magnitude of harm or discomfort anticipated in the research are not greater in and of themselves than those ordinarily encountered in daily life or during the performance of routine physical or psychological examinations or tests.

- Minimal Greater than minimal risk: Greater than minimal risk is a risk whereby the probability and magnitude of harm or discomfort anticipated in the research are greater in and of themselves than those ordinarily encountered in daily life or during the performance of routine physical or psychological examinations or tests. 


\section{APPENDIX II. Relations.}

Now that we have defined a number of universals in the domain of biomedical ethics, it is possible to organize these universals into a taxonomic structure using is_a:

- Researcher role is_a worker role [that inheres in a person and realized through a process of investigating and seeking to explain and predict some aspect of reality].

- Clinical trial researcher role is_a researcher role.

- Study subject role is_a study participant role [that inheres in an entity and is realized by the implementation of: (1) an observation design specifying how the entity is to be observed, and/or (2) an intervention design specifying the protocols applied to anything participating in the study, whether they are treated or acting as control].

- Human subject role is_a study subject role [that inheres in a person].

- Clinical trial performance (clinical trial) is_a process.

- Clinical trial drug role is_a test substance role [that inheres in a material entity-bearing drug role].

- Clinical trial device role is_a test substance role [that inheres in a device].

- Review is_a process.

- Clinical trial review is_a review.

- Expedited clinical trial review is_a clinical trial review.

- Full clinical trial review is_a clinical trial review.

- Organization is_a continuant entity [that can play roles, has members, and has a set of organization rules. Members of organizations are either organizations themselves or individual people. Members can play specific organization member roles that are determined in the organization rules. The organization rules also determine how decisions are made on behalf of the organization by the organization members.].

- Board is_a organization.

- Regulatory role is_a role [realized by the act of regulating or the state of being regulated].

- A regulator role is_a regulatory role [realized through the process of ensuring compliance with laws, regulations, and established rules].

- Role of institutional review board (IRB) is_a regulator role [that inheres in an organization].

- Role of IRB member is_a regulator role [that inheres in a person].

- Role of IRB chairperson is_a regulator role [that inheres in a person].

- Document is_a narrative object.

- Protocol document is_a document.

- Consent form is_a document.

- IRB review form is_a document.

- IRB submittal form is_a document.

- IRB decision letter is a document.

- Duty is_a principle.

- Ethical duty is_a duty.

- Beneficence is_a ethical duty.

- Non-maleficence is_a ethical duty.

- Justice is_a ethical duty.

- Ethical duty performance is_a process.

- Risk is_a disposition.

- Minimal risk is_a risk.

- Greater than minimal risk is_a risk.

- Specified_output role is_a role.

Now we can begin transforming our taxonomy into a domain ontology by linking universals with the relations part_of, preceded_by, has_participant, and inheres_in.

part_of relations:

- Start/end of clinical trial part_of clinical trial.

- Start/end of clinical trial review part_of clinical trial review.

preceded_by relations:

- Clinical trial preceded_by clinical trial review.

has_participant relations:

- Clinical trial review has_participant IRB.

- Clinical trial review has_participant IRB submittal form.

- Clinical trial review has_participant protocol document.

- Clinical trial review has_participant consent form.

- Clinical trial review has_participant IRB review form.

- Clinical trial has_participant clinical trial object. 
APPENDIX II. Continued.

- Clinical trial has_participant clinical trial researcher.

- Clinical trial has_participant human subject.

inheres_in relations:

- Risk inheres_in human.

- Clinical trial researcher role inheres_in person.

- Human subject role inheres_in human.

- IRB member role inheres_in person.

- IRB chairperson role inheres_in person.

Because this is a biomedical ethics ontology, the question arises as to the status of ethical duties as they exist between and among the participants in clinical trials and clinical trial reviews. Ethical duties are owed to persons, other human beings, and animals with fairly complex nervous systems, so we have chosen the following as representative of these relationships:

- is_owed: A relationship between persons whereby the person owed has a claim against those who fail to abide by some duty.

- owes_a: A relationship between persons whereby the person owing has a duty to perform some action or intended action to the person owed.

Thus, for example:

- Human subject is_owed ethical duty.

- Clinical trial researcher owes_a ethical duty.

Further, the end result of a clinical trial review is a decision letter, so we have chosen has_output to represent this relationship, as in:

- Clinical trial review has_output decision letter.

In fact, there exists a definition for has_output in the Open Biomedical Ontologies (OBO) Foundry

(http://purl.obofoundry.org/obo/), and we are following that definition here:

- has_output: A relationship between a protocol application and a participant in the protocol application. The output is, at a minimum, present at the end of the protocol application, but not all participants present at the end of the protocol applications are output, only those that are viewed as outputs according to the plan, and hence realize "output role."

Also, we have chosen to add a has_role relation, as in:

- Institutional review board (IRB) member has_role IRB.

- IRB chairperson has_role IRB.

Here, we define has_role as:

- has_role: A relationship between two independent continuants A and B in a particular context such that A realizes its role in B (see http://purl.obofoundry. org/obo/).

Besides IRBs, a person has_role in any number of organizations, like companies, military forces, civil interest groups, clubs, and others. Also, it is possible that an entire organization has_role another organization. For example:

- IRB at University at Buffalo has_role University at Buffalo.

- Illinois Antique Auto Club Chapter 1 has_role Antique Auto Club of America.

- USA has_role United Nations.

This relationship is not the same as part_of which, as we noted in the last chapter, has the property of being transitive (as well as reflexive and antisymmetric). Given this transitive property, with the part_of relationship, we are able to reason that if $A$ part_of $B$

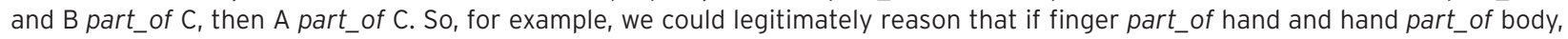
then finger part_of body. However, note that we cannot reason that if John has_role some organization $\mathrm{A}$ and that organization $\mathrm{A}$ has_role organization $B$, then John has_role organization B. John might have a role in the karate club, and the karate club might have a role in the Japanese club, but that does not mean that John has a role in the Japanese club. 